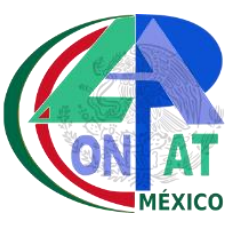

\title{
PRESERVACIÓN DE LA FACHADA DE UNA CONSTRUCCIÓN EXISTENTE PARA SU INCORPORACIÓN A UNA NUEVA EDIFICACIÓN EN ALTURA
}

\author{
R. HUSNI, P. MAYO, C. MACCHI, A. MALVAR PERRIN \\ Departamento de Construcciones y Estructuras, Facultad de Ingeniería UBA. Avda. Las Heras 2214 CABA. Argentina. \\ Estudio de Ingeniería C. Macchi, R. Husni y Asociados. Azcuénaga 1767 EP CABA. Argentina
}

\begin{abstract}
RESUMEN
El trabajo forma parte del proyecto y construcción de un edificio en torre para uso residencial y comercial de $24.0000 \mathrm{~m} 2$ con 2 subsuelos, ubicado en una cotizada zona de la Ciudad de Buenos Aires. En el predio existía una antigua construcción de arquitectura industrial inglesa de aproximadamente 100 años de antigüedad, conocida con el nombre de "La Imprenta", con estructura metálica, entrepisos de bovedilla y fundaciones directas superficiales, que ya había sido adaptado en sus 3 niveles para uso como galería comercial. Por su valor patrimonial, $\operatorname{los} 40 \mathrm{~m}$ de fachada del edificio en esquina de ladrillo visto y de $11 \mathrm{~m}$ de altura, debían ser preservados íntegramente con absolutas restricciones a cualquier intervención del lado exterior. En la presentación se describe la metodología aplicada para mantenerlo estable y sin afectación, así como las sucesivas etapas realizadas para submurar y sostener de manera provisoria la fachada de ladrillo, construir las fundaciones y la nueva estructura de hormigón armado por debajo y a través de la estructura metálica interior existente, la que fue preservada para darle estabilidad al muro hasta que estuvo asegurado por la nueva construcción. Finalmente se detallan los procedimientos de control y los detalles constructivos para incorporar definitivamente el muro preservado a la nueva construcción.
\end{abstract}

Palabras Clave: Estructura provisoria; Preservación; Fachada

\begin{abstract}
The work it's part of a project and construction of a tower building for residential and commercial use; of $240.000 \mathrm{~m} 2$ with two basements, located in a highly valuable neighborhood of the city of Buenos Aires. Previously, in the site; there was an old construction of english industrial architecture of approximately 100 years old commonly known as "La Imprenta" (The Print); with a steel structure; direct superficial foundations and vaulted mezzanines, which had been adapted in their three levels to use as a commercially gallery. For its heritage value, the $40 \mathrm{~m}$ facade of the building in an exposed brick corner and $11 \mathrm{~m}$ high, should be preserved entirely with absolute restrictions to any intervention on the exterior side. The presentation describes the methodology applied to keep it stable and unaffected, as well as the successive stages carried out to sustain the brick facade, build the foundations and the new reinforced concrete structure below and through the existing structure metallic, which was preserved to give stability to the wall until it was secured by the new construction. Finally; we explain the control procedure and the constructive details for incorporating definitively the preserved wall to the new construction.
\end{abstract}




\section{INTRODUCCIÓN}

En la segunda mitad del siglo XIX las actividades relacionadas al hipismo imprimieron un carácter particular al sector de la ciudad, ubicado al pie de la barranca en los terrenos no urbanizados cercanos al Río de la Plata

En un edificio, de dos plantas con fachada de ladrillo visto de arquitectura industrial inglesa construido en la década de 1920, funcionó la imprenta del Jockey Club, ubicado en la esquina de las calles Maure y Migueletes del barrio de Palermo de la Ciudad Autónoma de Buenos Aires.

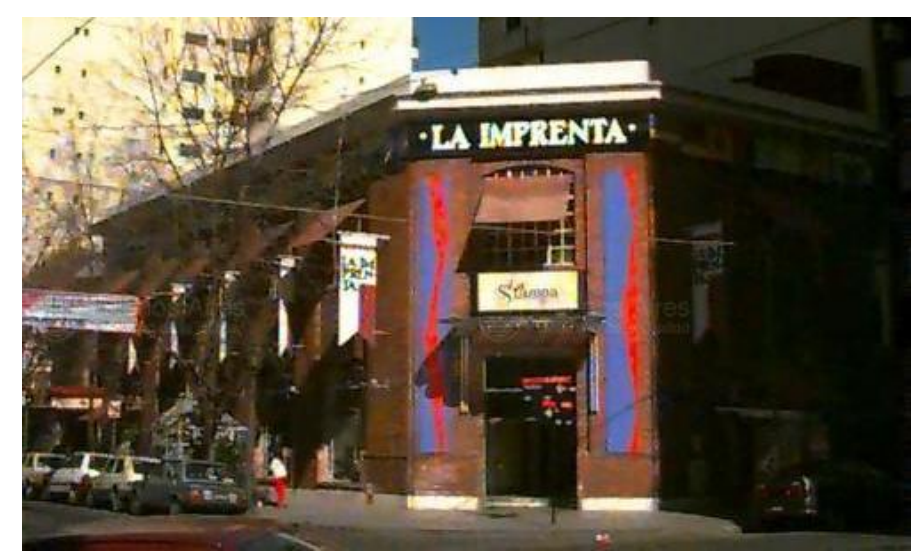

Figura 1- Fachada (circa 1980)

Se trataba de una construcción industrial de dos plantas, desarrolladas alrededor de un largo patio central que permitía el ingreso de luz hasta planta baja. La fachada sobre la línea oficial de ambas calles estaba formada por un muro de excelente calidad con molduras y salientes, de $50 \mathrm{~cm}$ de espesor de mampostería de ladrillos, con amplias aberturas dispuestas en forma regular.

La dinámica del crecimiento urbano permitió la revalorización de esos terrenos estratégicamente ubicados, por lo que en 1982 se cambió el destino industrial del edificio, que, integrado a antiguas caballerizas recicladas y a otros predios contiguos, conformó un amplio complejo comercial que tomó el nombre de "La imprenta", Figura 1.

En intervenciones posteriores se realizaron diversas modificaciones, cegando el patio central sobre planta baja y agrandado el ancho del acceso principal en la ochava de la esquina con puede apreciarse en la Figura 2.

En los últimos años el auge de construcción de edificios de alta categoría y grandes torres en esa zona generó las condiciones para desarrollar en ese particular terreno de $1.700 \mathrm{~m} 2$ un proyecto residencial y comercial de $24.000 \mathrm{~m} 2$ cubiertos.

Al comienzo de esta década, ante el cierre del centro comercial, la firme disposición conservacionista, impuso como condición para poder desarrollar la nueva construcción, la preservación de la fachada, integrándola al nuevo proyecto arquitectónico.

La construcción debía desarrollarse cuidando especialmente la fachada y sus componentes lo que requería trabajar con precauciones excepcionales que condicionaron el proyecto y su posterior materialización.

Hubo que preservar íntegramente el muro de frente sobre las calles Maure y Migueletes en sus $40 \mathrm{~m}$ de extensión y casi $11 \mathrm{~m}$ de altura, sin alterar ningún elemento, fuera este original o producto de alguna intervención anterior.

Se descartaron las instalaciones y apuntalamientos provisorias del lado exterior sobre la vereda, debido a su escaso ancho y a las instalaciones de servicios que se encontraban debajo de la calzada. Se mantuvieron las carpinterías con sus vidrios, solamente se dispuso el cerco de protección de obra junto a la línea oficial. 


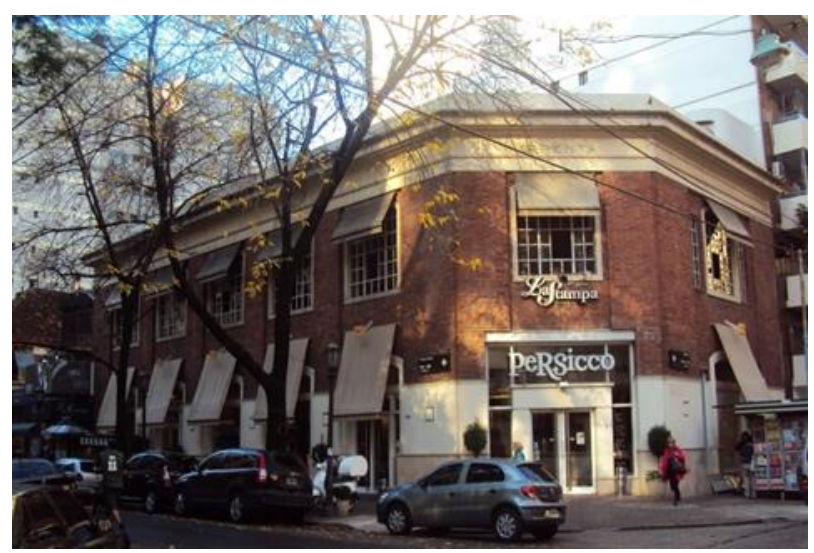

Figura 2. La Imprenta - Fachada (circa 2010)

El proyecto arquitectónico se adaptó a los condicionantes geométricos y estéticos de la fachada.

\section{DESCRIPCIÓN DEL EDIFICIO EXISTENTE}

Como no se disponía documentación técnica de la construcción existente, se determinaron mediante cateos las dimensiones y el estado de los elementos estructurales, detectando que los muros y las columnas se encontraban fundados en una zapata de ladrillos. Para el caso del muro del frente con forma de pilastra continua, cuyo talón alcanzaba un ancho de $1 \mathrm{~m}$ a -2,40m de profundidad. Figura 3

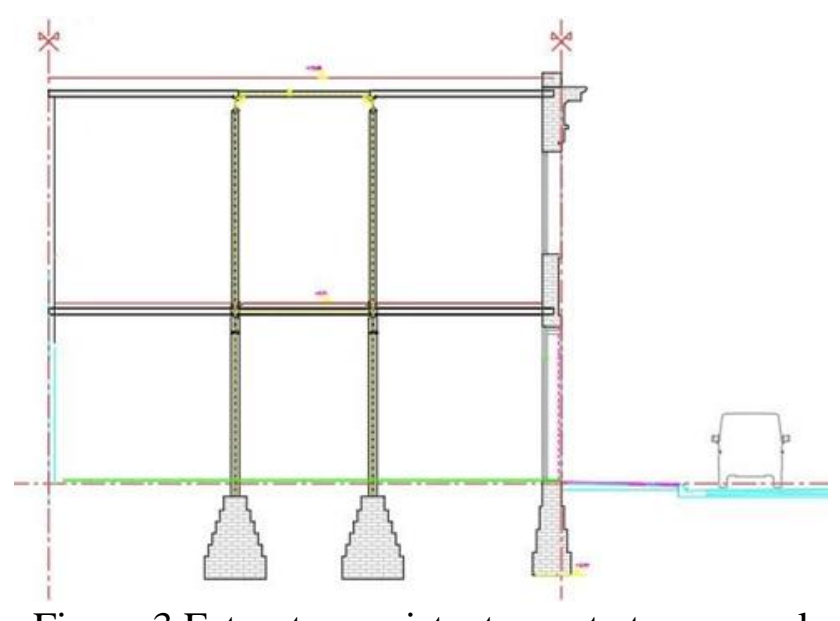

Figura 3 Estructura existente; corte transversal

Las 8 columnas estaban fundadas en pilastras piramidales que alcanzaban la medida de 1,60m x 1,60m a $-2,50 \mathrm{~m}$ de profundidad.

El sistema estructural metálico, como se ve en las Figura 3 y 4, 5 y 6, estaba constituido por 8 columnas centrales con vigas principales doble $\mathrm{T}$ de $510 \mathrm{~mm}$ en el primer piso y de $385 \mathrm{~mm}$ en el segundo. Los forjados eran entrepisos en bovedillas simples de ladrillos con perfiles IPN 180, que apoyaban en los muros perimetrales y en el marco estructural central. 


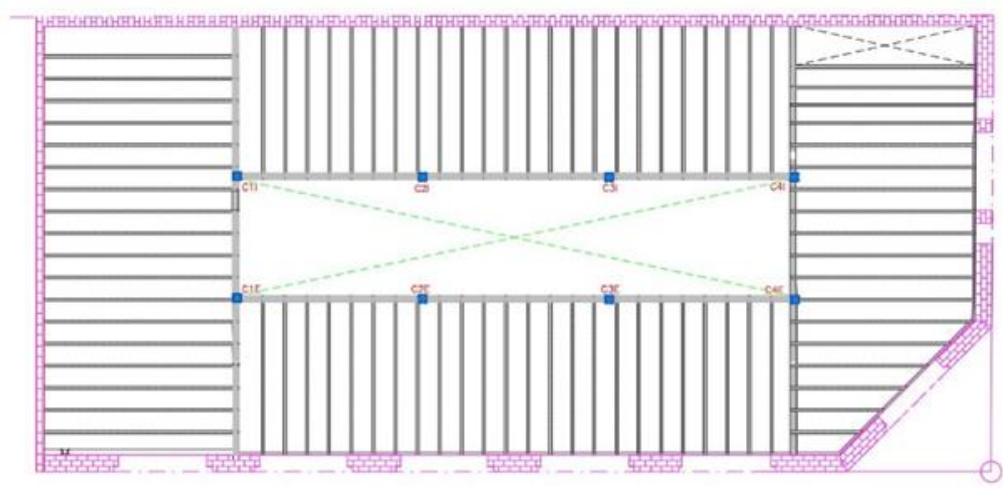

Figura 4. Estructura original; Planta
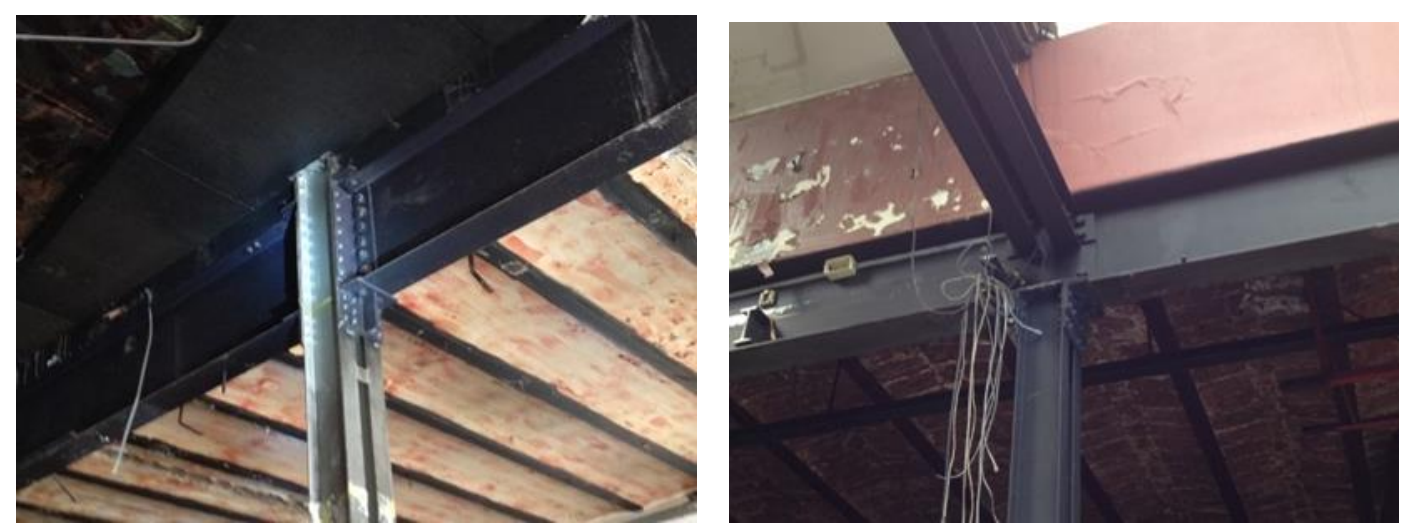

Figura 5 y 6- Estructura original; Forjados, vigas y columnas

\section{NUEVO PROYECTO ARQUITECTÓNICO}

Limitando la descripción a la zona de interés, los 13,50 m de frente sobre la calle Maure y los $28 \mathrm{~m}$ de frente sobre la calle Migueletes, se proyectó un subsuelo de 4,00 m de altura y fundaciones a $6 \mathrm{~m}$ de profundidad. En los $30 \mathrm{~m}$ restantes con frente a Migueletes, el proyecto contemplaba dos subsuelos destinados a estacionamiento vehicular.

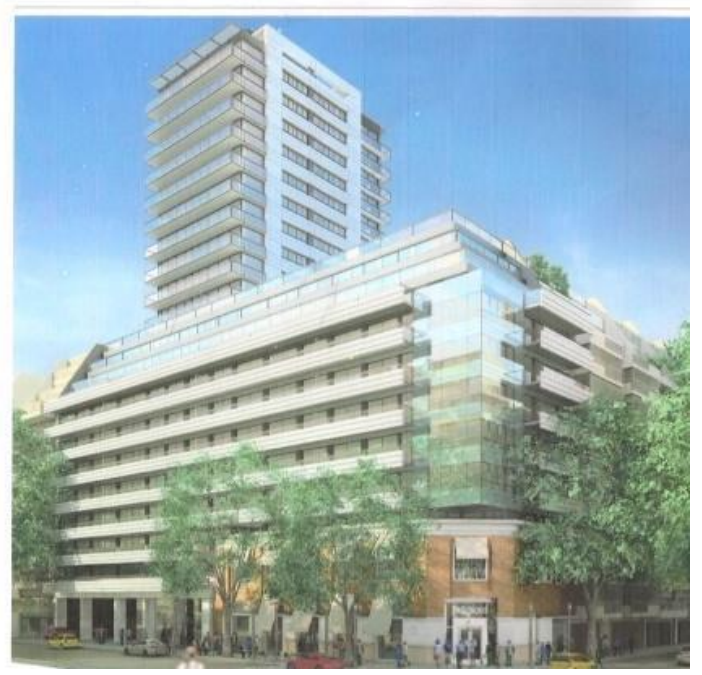

Figura 7. Perspectiva del proyecto 
La planta baja, el entrepiso $(\mathrm{NSL}=+3.05)$ y primer piso $(\mathrm{NSL}=+5,70)$ del Sector esquina se destinaron a locales comerciales con doble altura en el frente de la planta baja, para compatibilizarlo con las aberturas existentes. El coronamiento del muro de mampostería (NIVEL. +10,60) pasa a constituirse en el antepecho de los balcones de la segunda planta (NSL=+9,12). Los niveles de las nuevas plantas no coincidían con el nivel de la estructura de los forjados preexistentes. El basamento del edificio se completa con planta tipo desde $3^{\circ}$ a $8^{\circ}$, retiros progresivos de $9^{\circ}$, a $11^{\circ}$ y $12^{\circ}$ destinada a equipos y salas da máquinas.

Adosada a la zona de nuestro interés y retirada 8,00m de la línea municipal se construyó una torre de $16 \mathrm{~m}$ x 26m de planta con 25 Pisos de altura

\section{ESTRATEGIA CONSTRUCTIVA ADOPTADA}

El objetivo era construir la estructura del nuevo edificio, desde sus fundaciones por detrás del muro sin que este sufriera ningún daño, para lo que era necesario liberar el espacio existente por detrás y por debajo de la fachada.

Por las restricciones para poder instalar estructuras transitorias del lado exterior, la estabilidad del muro debía asegurarse desde el interior del predio.

Luego de analizar distintas alternativas se decidió utilizar la estructura metálica existente como estructura de arriostramiento provisoria, capaz de impedir el volcamiento de la fachada hasta que la nueva estructura de hormigón armado la pudiera reemplazar en esta función.

Para poder atravesar las plantas existentes con la nueva estructura de hormigón armado debían eliminarse las losas de la bovedilla, al igual que la fachada lateral interna que completaba el rectángulo cerrado del muro perimetral.

En esas condiciones el entramado de vigas y columnas metálicas resultante resultaba lábil y debía ser rigidizado en las dos direcciones para soportar las acciones horizontales, tales como el viento, excentricidades constructivas, etc.

La estructura definitiva de hormigón del edificio debía desarrolarse coexistiendo con el entramado metálico de arriostramiento.

La construcción por debajo requería excavar manteniendo la sustentación de la estructura metálica mediante una estructura de transición y la submuración de los muros de fachada, restringiendo el desplazamiento horizontal de la estructura de apeo y soportando el empuje del suelo exterior a la excavación. Figura 8.

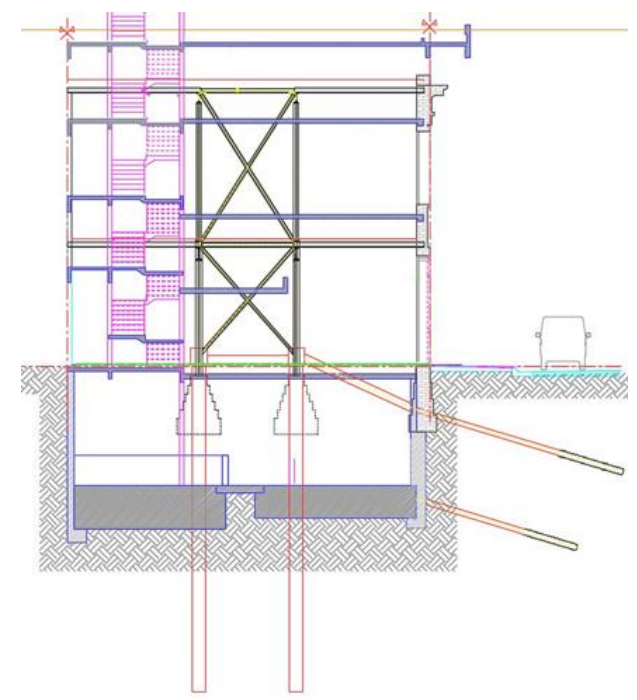

Figura 8. Estructura existente+ transitoria + definitiva corte transversal 


\section{PROCEDIMIENTO CONSTRUCTIVO}

\section{1 Enmarcado de vanos}

Antes de comenzar las tareas relacionadas con las estructuras provisorias y de excavación, se colocaron marcos internos de gran rigidez, con tirantes de madera de 3'x 6', a efectos de imposibilitar cualquier deformación de los vanos del muro de fachada en cuestión. Figura 9

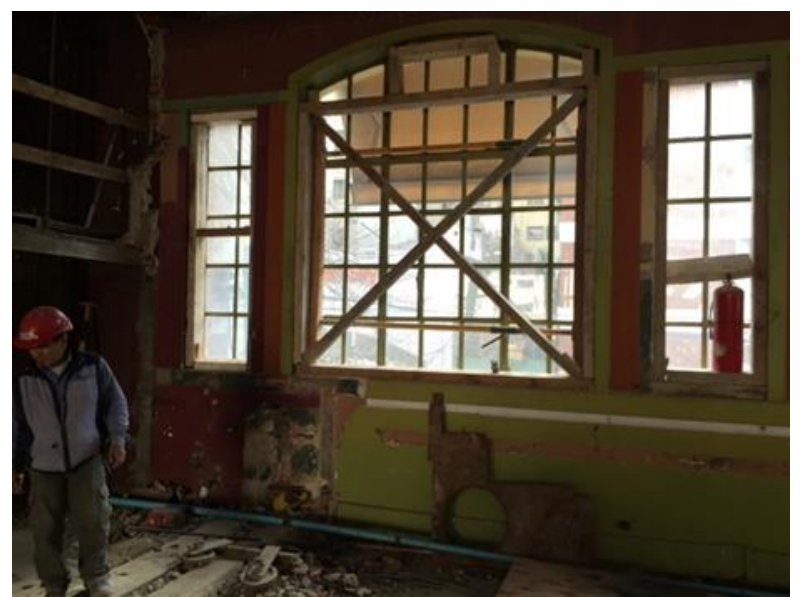

Figura 9. Vista de enmarcado de vanos

\section{2 Rigidización}

Para poder desarrollar los trabajos debían demolerse todos los muros interiores y las losas de las bovedillas, con lo que la construcción perdía su capacidad de trasladar las acciones horizontales. Para resolverlo se dispusieron cruces horizontales soldadas a los perfiles de bovedilla y cruzando el patio central.

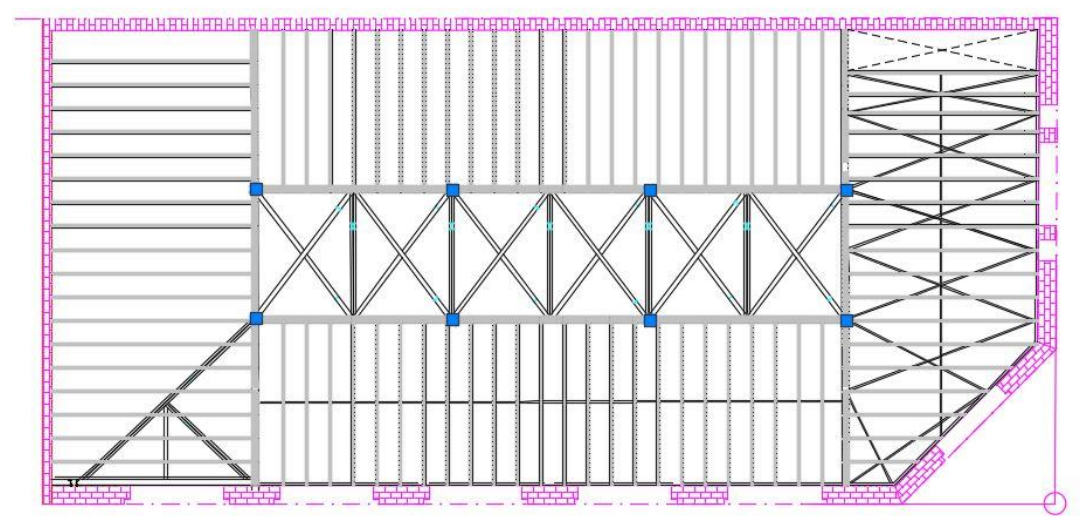

Figura 10. Estructura original rigidizada en su plano; Planta

A fin de rigidizar el sistema vigas y columnas se procedió al montaje de cruces de San Andrés metálicas, soldadas a la estructura existente de manera transversal y longitudinal vinculando las columnas del sistema. Figuras 10, 11 y 12 

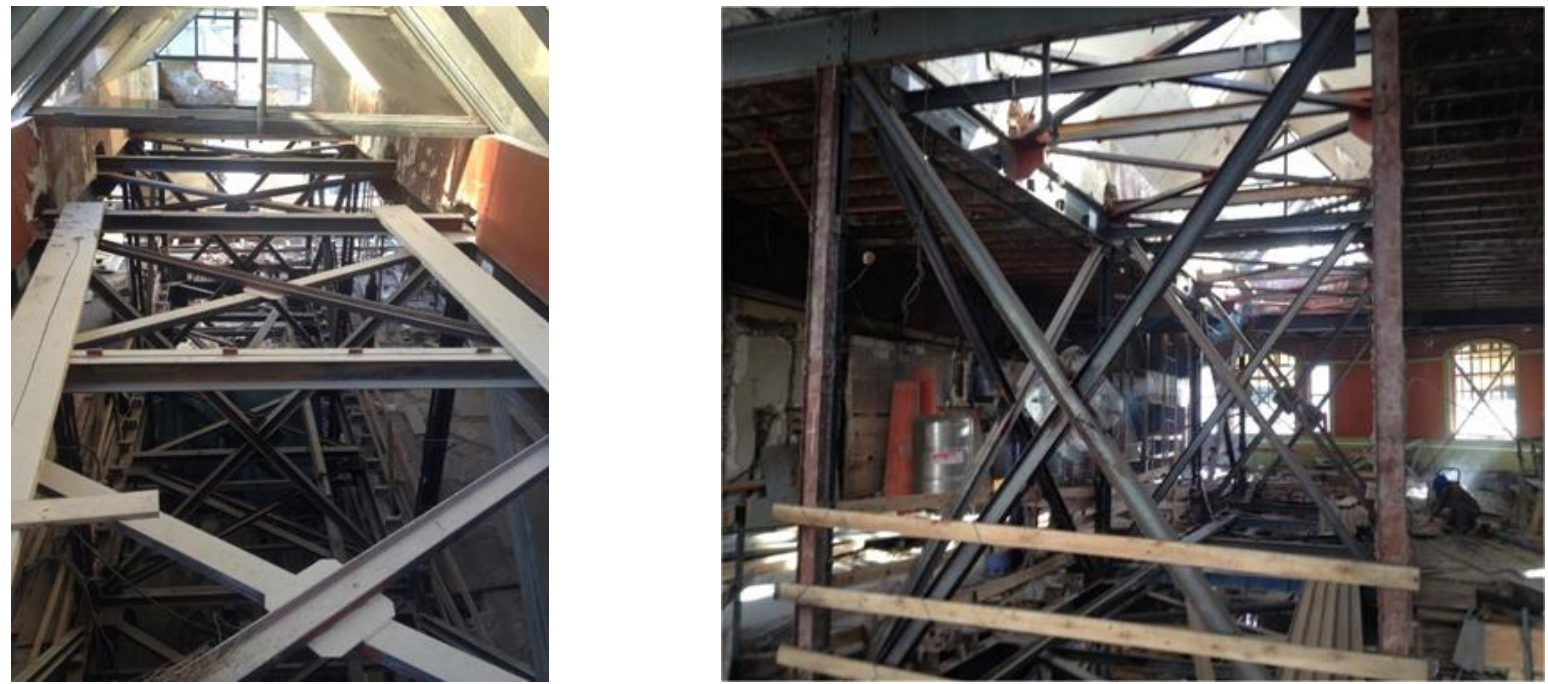

Figuras 11 y 12. Vistas de la cruces e San Andrés horizontales y verticales

Para demoler la fachada lateral interna se desmontó el paño extremo de forjado que apoyaba en ella y fue necesario arriostrar el borde libre extremo de fachada que quedaba en voladizo. Figuras 13 y 14.
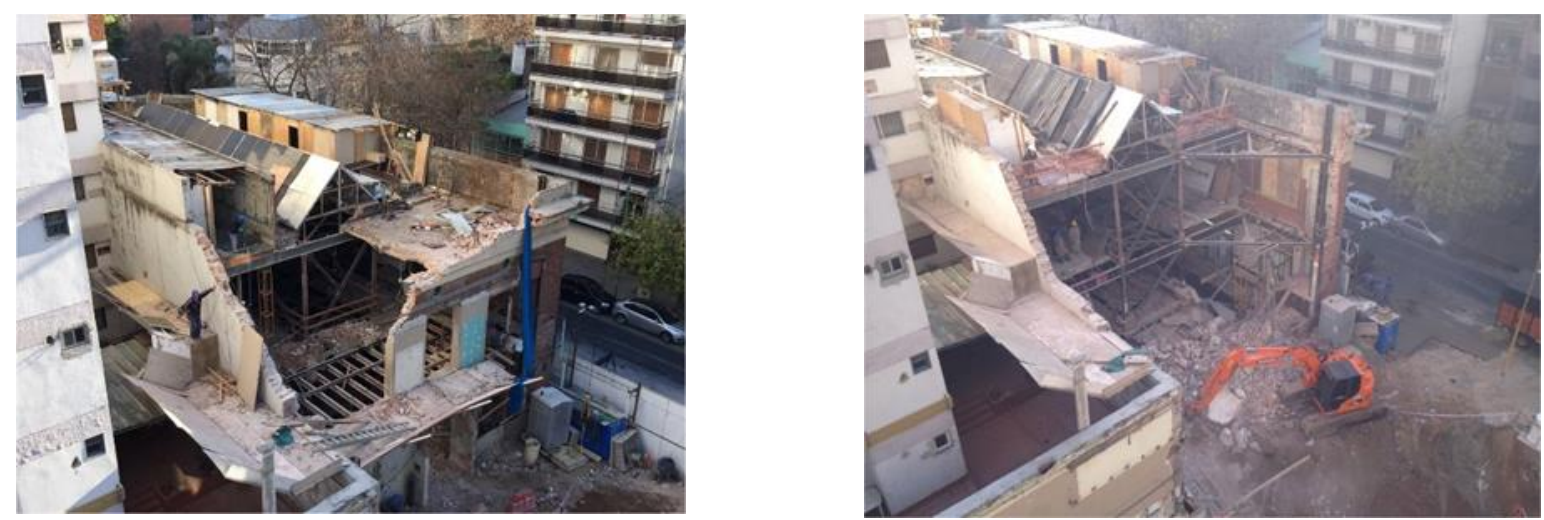

Figuras 13 y 14. Vistas de la demolición del paño extremo y arriostramiento de la fachada

Como se ve en las figuras mencionadas se mantuvo la cubierta superior a los efectos de realizar todos los trabajos protegidos de las lluvias.

\section{3 Ejecución de fundaciones transitorias mediante pilotes}

Para el recalce de la estructura existente, era necesario profundizar el plano de fundación para lo que se ejecutaron 2 hileras de 5 pilotes de $50 \mathrm{~cm}$ de diámetro y $12 \mathrm{~m}$ de profundidad, alineados con las columnas existentes, encamisados en la parte superior debido a las características del suelo a nivel superficial, Figura 15

\section{4 Construcción de las vigas de apoyo de las columnas existentes}

A nivel +0.60 se construyeron 2 vigas longitudinales de $60 \times 60$ apoyadas en la línea de los 5 pilotes mencionados, para constituir el nuevo soporte de las columnas metálicas, lo que permitiría eliminar sus bases. Figura 16. Como las columnas atraviesan las vigas, para la transferencia de las cargas de las estructuras existentes a la nueva transitoria, se utilizaron pernos soldados Studd Nelson, localizados en la altura de la nueva viga de apoyo. 

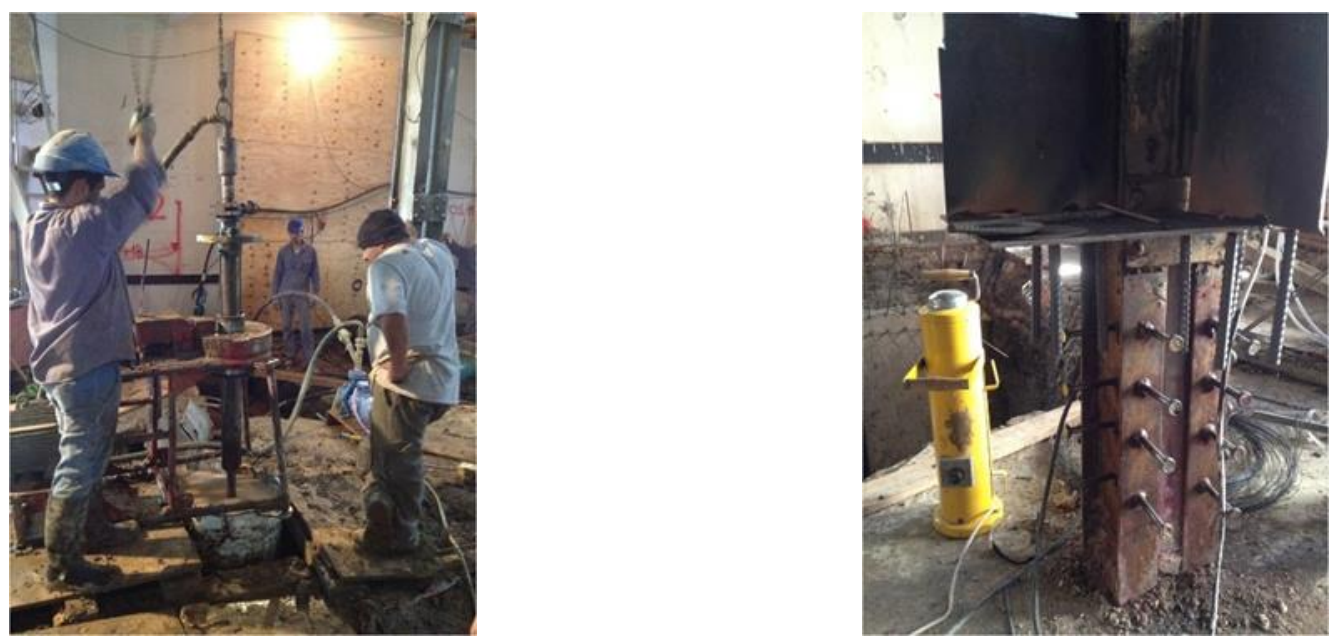

Figuras 15 y 16. Ejecución de pilotes y pernos de transferencia de cargas

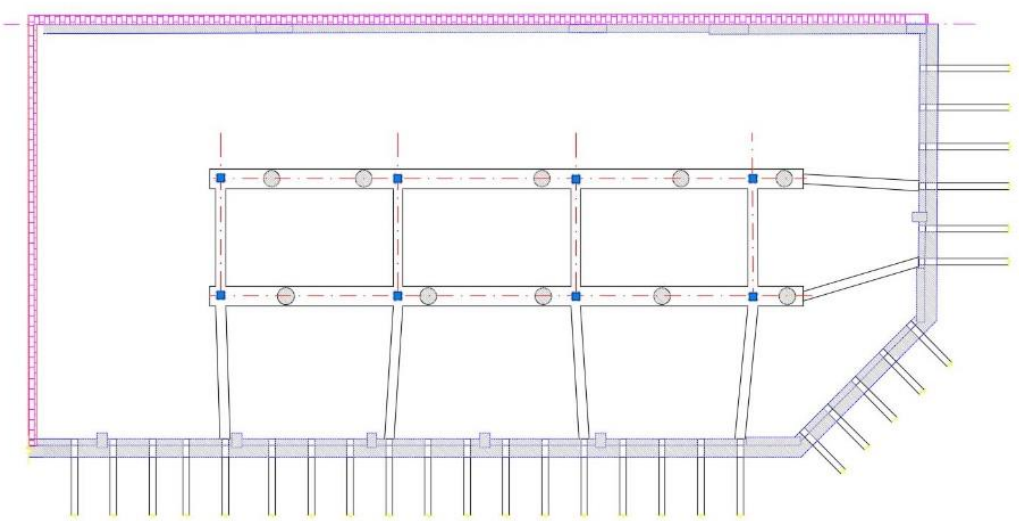

Figura 17 - Estructura de apeo - Planta a nivel +0.60m

En las Figuras 18 se visualiza el encofrado de una de las nuevas vigas de apoyo de las columnas y en la Figura 19 las vigas ya hormigonadas, con las columnas que la traviesan, transfiriéndole sus cargas, del orden de las 40 ton a través de los pernos soldados localizados en el interior de las vigas y en un tramo de columna encamisada
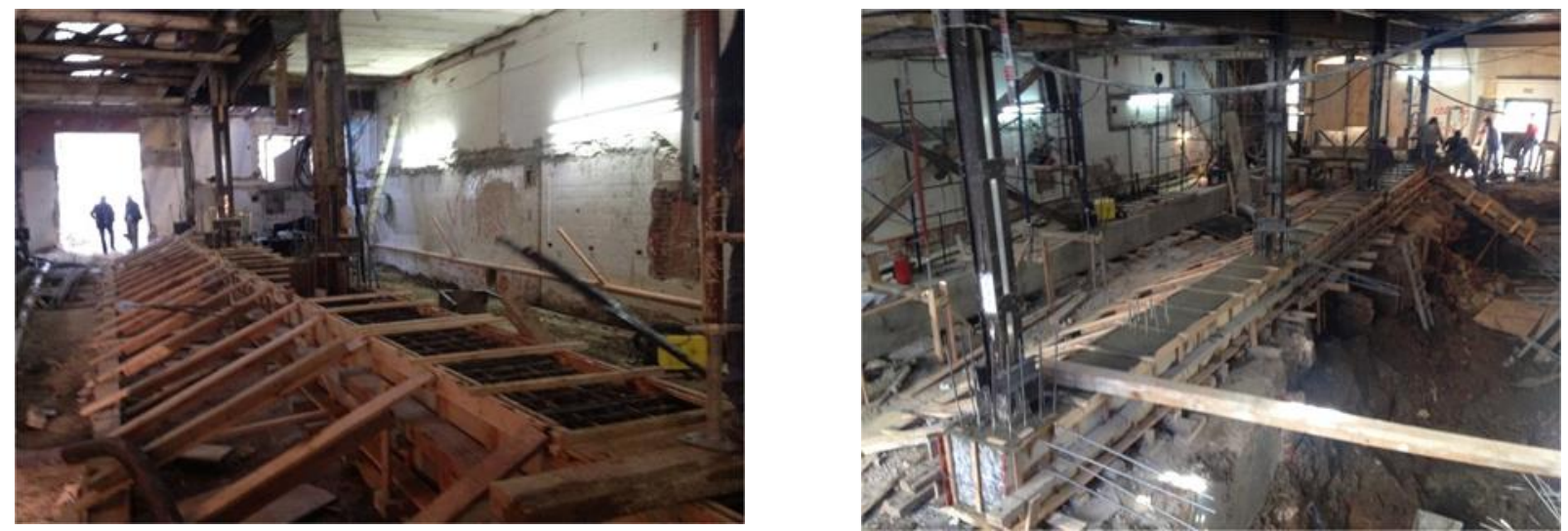

Figuras 18 y 19. Vista de la estructura de apeo a nivel $+0.60 \mathrm{~m}$ 


\section{5 Primer fase de excavación y ejecución de anclajes}

En la primera etapa de excavación por pocetes se llegó al nivel $-2,40 \mathrm{~m}$ coincidente con el nivel de apoyo del muro, perfilando la mampostería al plano vertical interior. Figura 20. Se ejecutaron anclajes tipo IRC con una inclinación de 30 grados perforando el muro de fachada y con desarrollo hacia el sector de calle evitando posibles interferencias con las instalaciones bajo la vereda.

En coincidencia con el muro de mampostería, se dispuso en cada anclaje un cabezal troncocónico que facilitaba la transferencia de la carga del empuje de tierra que soportaba el muro al anclaje. Figura 21 En esta etapa comenzó también el control de la verticalidad del muro, el que se realizaba semanalmente. A tal efecto se había relevado la verticalidad inicial en distintos sectores del muro, el que previamente al comienzo de los trabajos, desplomes de distinta magnitud.

Esta excentricidad inicial fue considerado en los cálculos de la estabilidad del muro
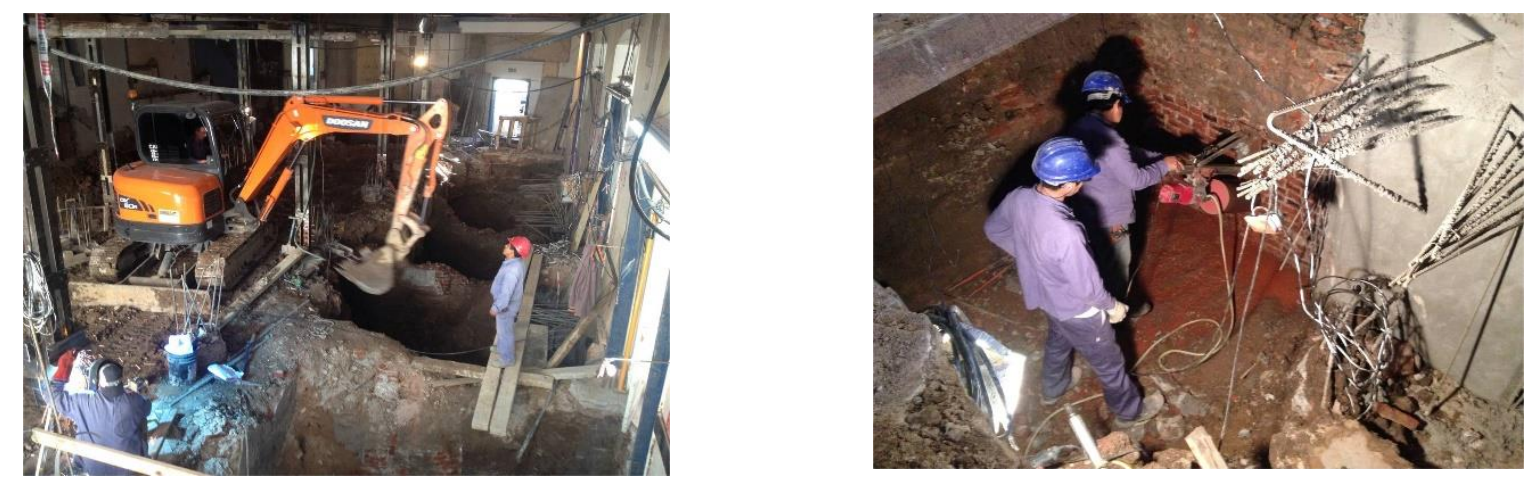

Figuras 20 y 21. Primera fase de excavación y realización de anclajes

\section{6 Realización de puntales inclinados y primer nivel de tabique}

Se construyeron puntales/tensores inclinados de hormigón armado que iban desde las cabezas de los micropilotes de anclaje hasta los puntos de apeo de la estructura en la dirección transversal y longitudinal.

De este modo se reemplazaba la condición de estabilidad horizontal que el suelo retirado brindaba al muro y, a su vez proporcionaba puntos fijos al reticulado de la estructura existente a nivel de las fundaciones. Figuras 17, 22 y 23. Se completaba la tarea realizando progresivamente con hormigón proyectado un tabique de $20 \mathrm{~cm}$ de espesor desde el nivel de la futura losa sobre primer subsuelo (NSL=0.27 ) hasta $-2,40 \mathrm{~m}$, para cumplir con funciones portantes, de contención de suelo y estanqueidad por la presencia de la napa freática
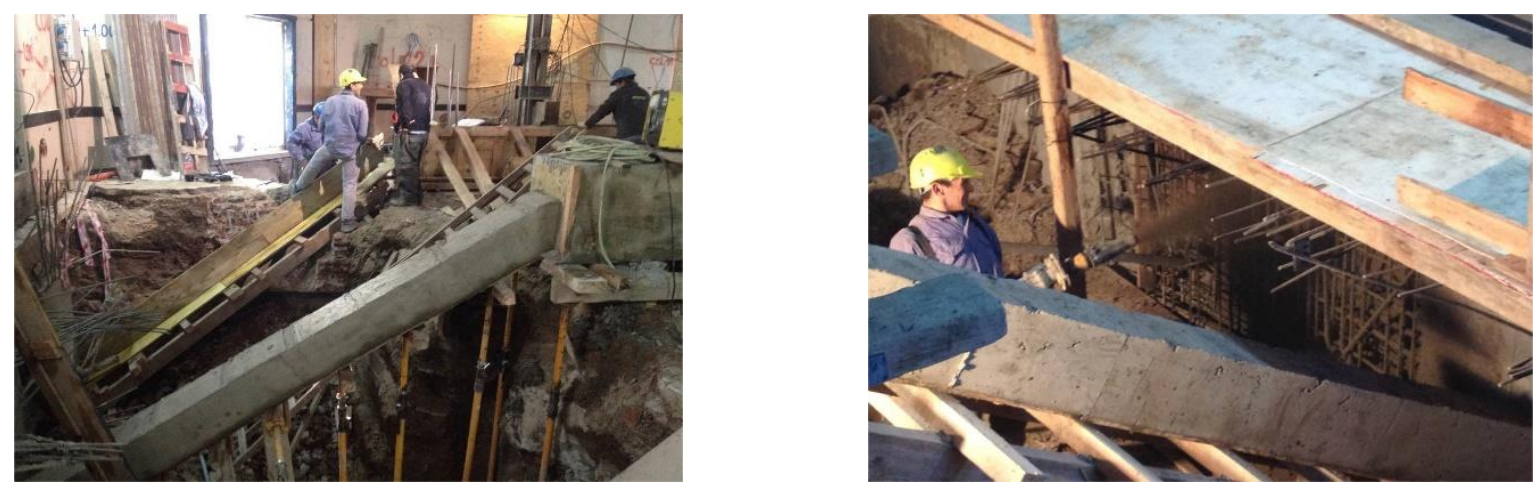

Figuras 22 y 23. Vista de puntales inclinados y hormigonado de los tabiques perimetrales 


\section{7 Segunda fase de excavación y demolición de bases}

Una vez garantizada la estabilidad horizontal del muro de fachada y del marco de apeo con la ejecución de todos los anclajes de la hilera superior, los puntales y la estructura de recalce, se procedió a completar la excavación de toda la planta hasta alcanzar el nivel -2,40. Figura 24

Posteriormente, ya con acceso para equipos dotados de martillos neumáticos, se procedió a demoler las bases de la estructura metálica, Figura 25, con lo que la estructura superior pasó a transferir las cargas de las columnas metálicas a través de las vigas de apeo en los pilotes actuando ahora como nuevas fundaciones.
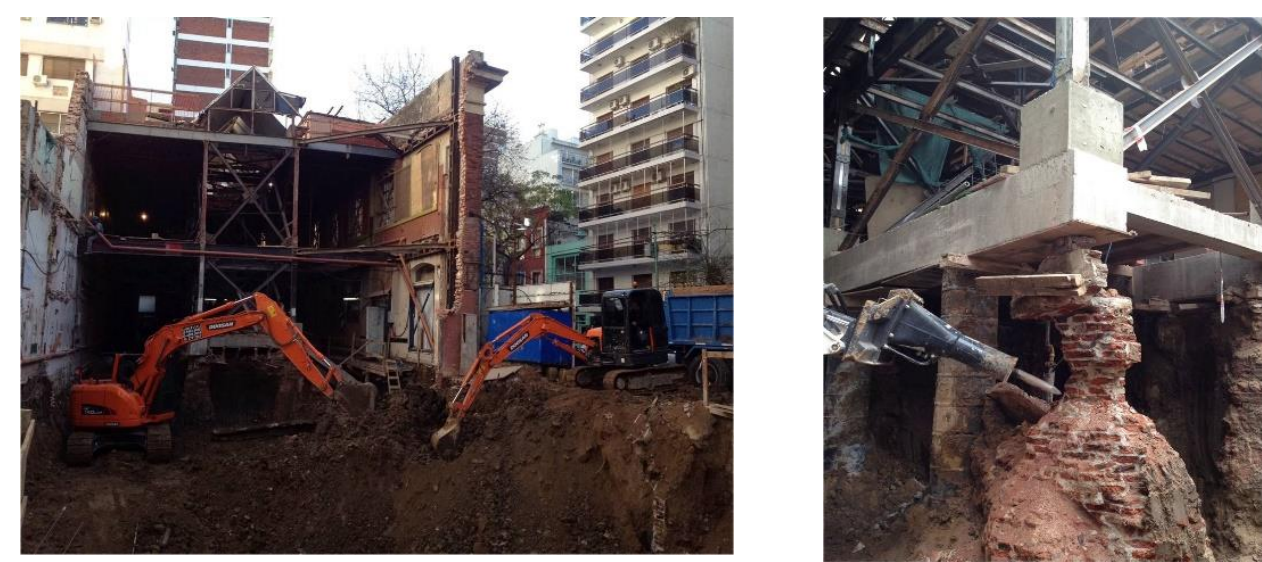

Figura 24 y 25. Segunda fase de excavación y demolición de la bases

\subsection{Segundo nivel de excavación, ejecución de anclajes}

Se continuó con la excavación hasta el segundo nivel que correspondía al nivel de fundación de la estructura definitiva a $-6,00 \mathrm{~m}$.

La subumuración del muro de fachada se hizo siguiendo el régimen de troneras, ejecutando el perfilado manual, armado y gunitado de los tabiques perimetrales por debajo del nivel -2,40 con los anclajes y su zapata correspondiente. El espesor de los tabiques en este tramo inferior es de $50 \mathrm{~cm}$, enrasado con el perfil del tramo superior de $20 \mathrm{~cm}$ para constituir el apoyo del muro de fachada.

En las Figuras 26 y 27 se aprecian estados intermedios de finalización de los submurales y la tarea de retirar la tierra por debajo del nivel $-2,40$ hasta llegar a nivel de las fundaciones proyectadas para la nueva edificación.
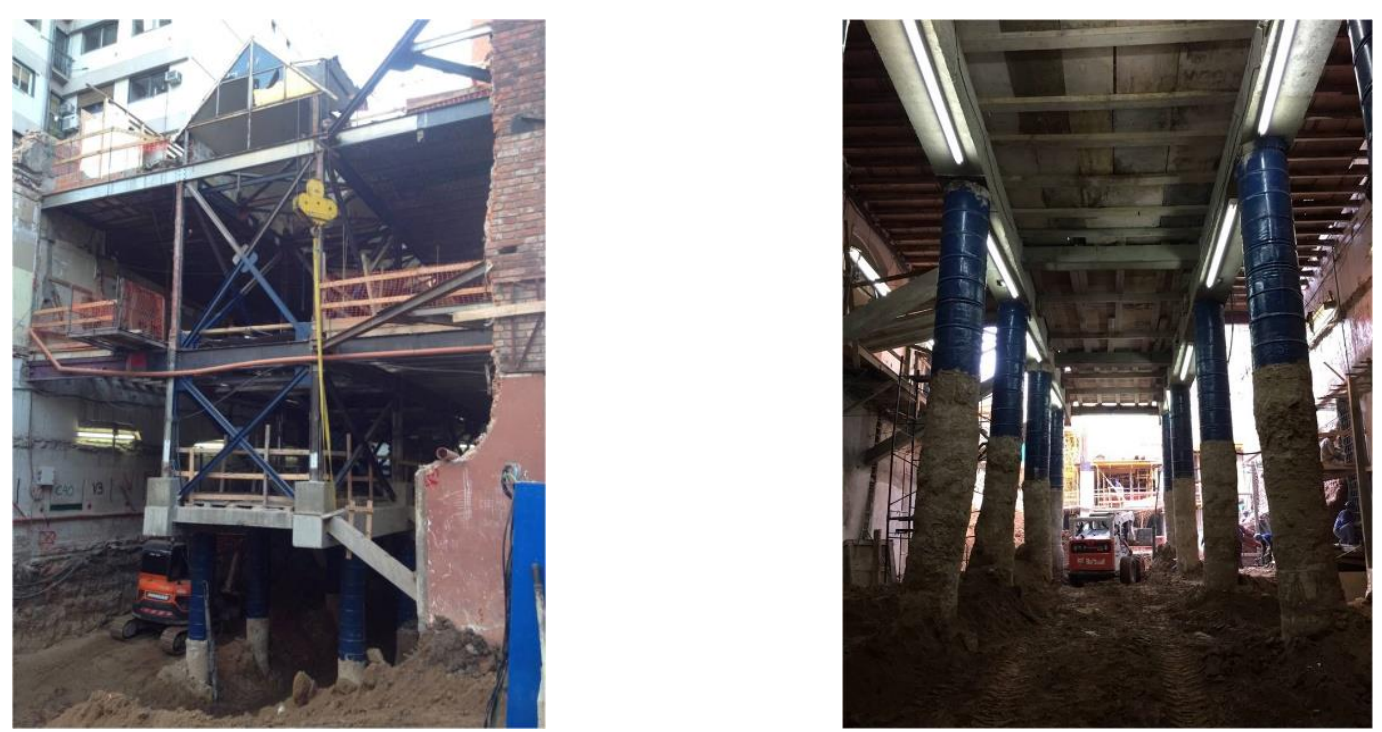

Figura 26 y 27. Vistas del segundo nivel de excavación 


\subsection{Ejecución de fundaciones del edificio}

Se ejecutaron las fundaciones definitivas que consisten en plateas y bases combinadas con alturas desde $1,20 \mathrm{~m}$ a $1,60 \mathrm{~m}$ asentadas entre $-5,60 \mathrm{~m}$ y $-6,00 \mathrm{~m}$ respectivamente. En los espacios entre las bases se construyó una losa de subpresión convenientemente anclada a las fundaciones. Cabe destacar que, aunque la geometría de la estructura de recalce fue definida de modo de sortear en lo posibles interferencias con las estructuras proyectadas para el edificio, esto no se pudo lograr en la totalidad de los casos para las fundaciones, por lo que en algunas situaciones algún tramo de pilote quedó incorporado a alguna de ellas. Figuras 28 y 29. El perímetro azul de los pilotes se corresponde con el sector en que fueron encamisados al momento de su construcción.
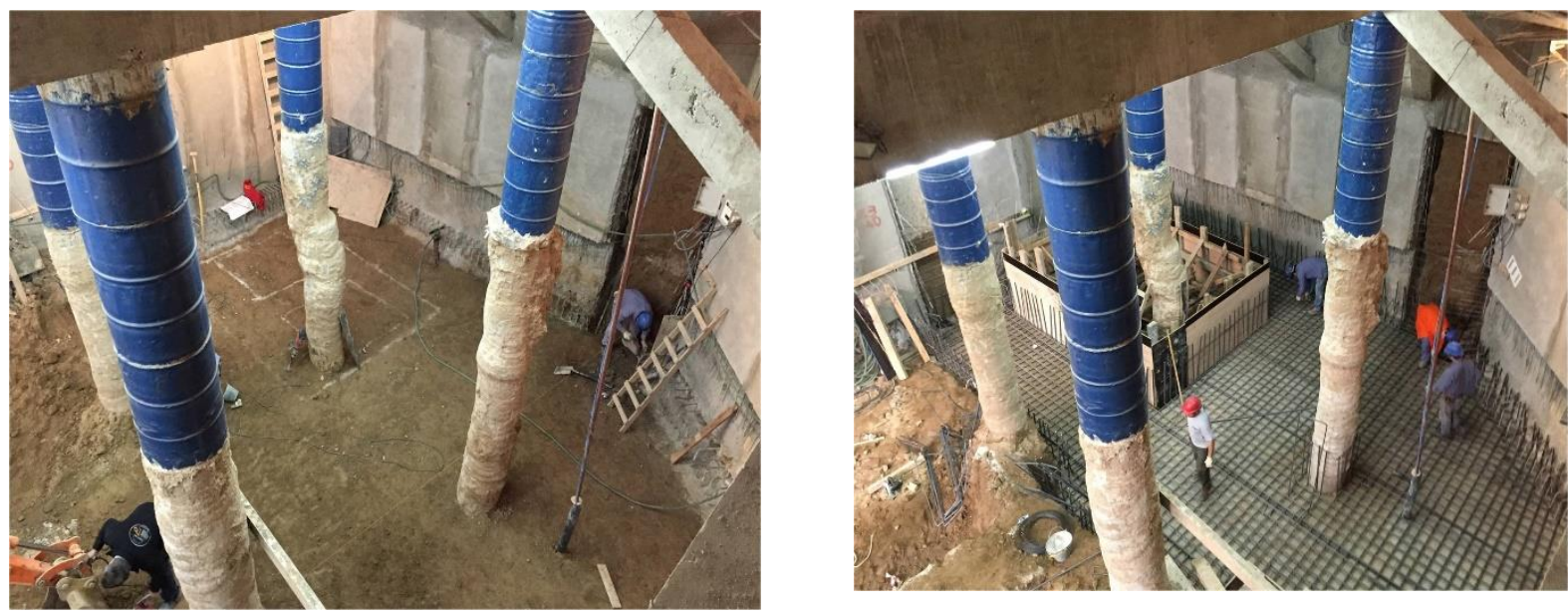

Figura 28 y 29. Vistas de la realización de las fundaciones de la zona de la esquina

En las Figuras 30 y 31 se aprecia el estado de la obra mientras se construían las fundaciones por debajo de la construcción preexistente.
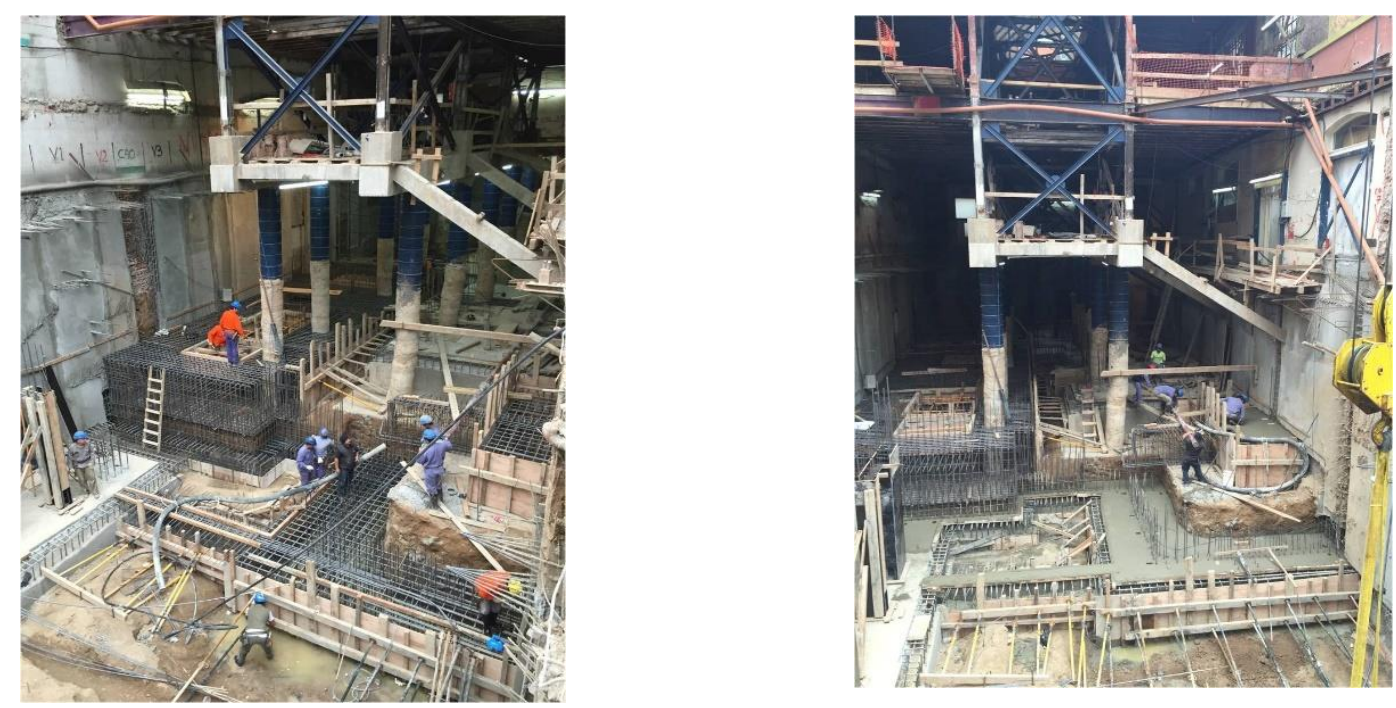

Figura 30 y 31 . Fundaciones del sector opuesto a la esquina

\subsection{Ejecución de columnas de subsuelos y losa de planta baja}

Con el volumen despejado entre las nuevas fundaciones y las vigas de hormigón transitorias, se realizaron los encofrados, las armaduras y la colocación del hormigón correspondiente a la losa sobre el subsuelo. Figuras 32 y 33 

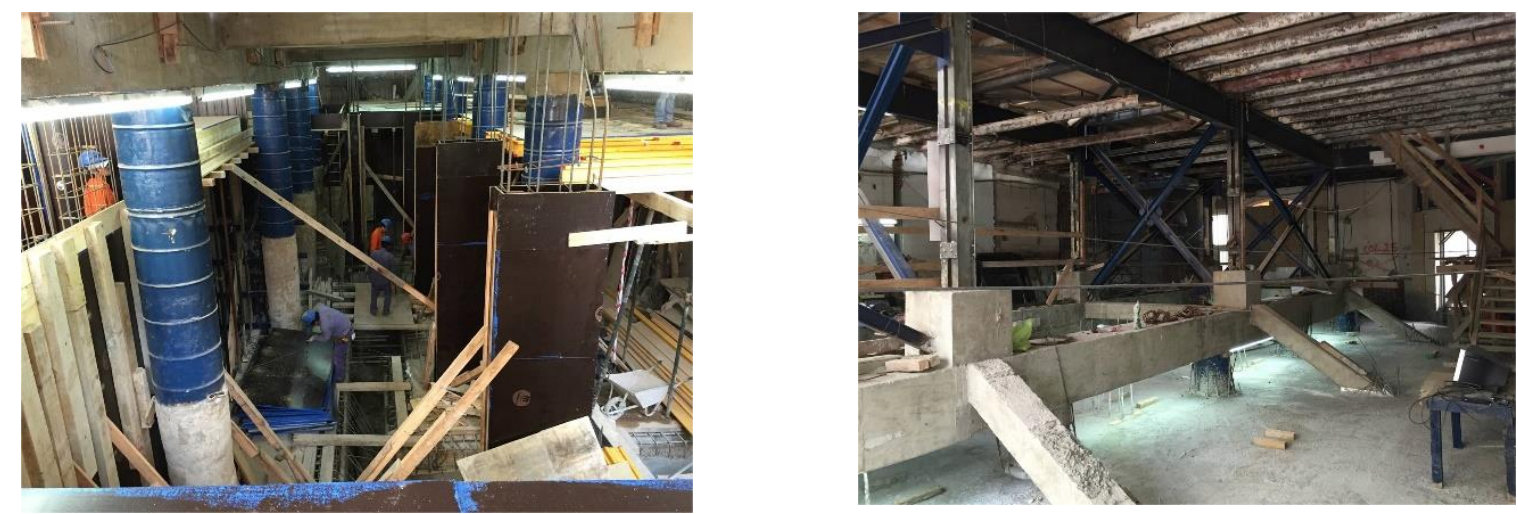

Figura 32 y 33 - Estructura sobre subsuelo en preparación y ya hormigonada

\subsection{Ejecución de columnas y losas de 1er, 2do y 3er piso}

Continuando con el mismo procedimiento, a medida que se construyeron las columnas y losas de los niveles de primer, segundo y tercer piso se realizaron los anclajes del muro de fachada a esta nueva estructura de manera que la vinculación a la estructura provisoria resulte innecesaria. Figuras 34 y 35.
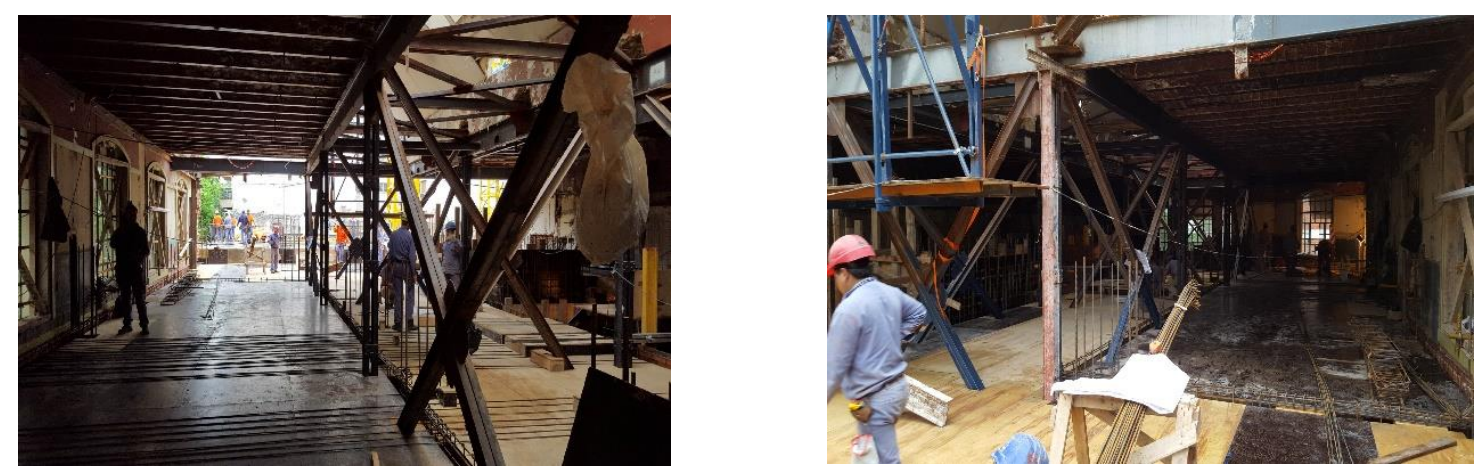

Figura 34 y 35 . Encofrado de estructura sobre entrepiso

Asimismo, se dejaron los pases necesarios en coincidencia con las columnas metálicas para ser cegados luego de desmontarlas

\subsection{Desmontaje de la estructura existente y demolición de estructura provisoria}

Una vez completada la estructura definitiva hasta el nivel de la losa de $2^{\circ}$ piso (Nivel $+9,12 \mathrm{~m}$ ) y verificados todos los anclajes de los muros de fachada, se procedió al desmontaje de la estructura metálica original y a realizar los arreglos de las interferencias con las nuevas estructuras.

Para finalizar, se demolieron todos los elementos estructurales que fueron útiles sólo al estado provisorio, esto es las vigas de apeo, sus riostras y los pilotes

\section{CONCLUSIONES}

La preservación de frentes de construcciones existentes cuando deben ser mantenidos para incorporarlos a nuevas edificaciones conllevan obras de sostenimiento importantes y complicaciones constructivas de distinto grado según puedan ser estabilizados desde el exterior o del interior de las obras.

En el primer caso, cuando es factible, implican grandes interferencias con la circulación de los peatones y del tráfico vehicular, pero se ve facilitada la operatoria dentro de la obra. 


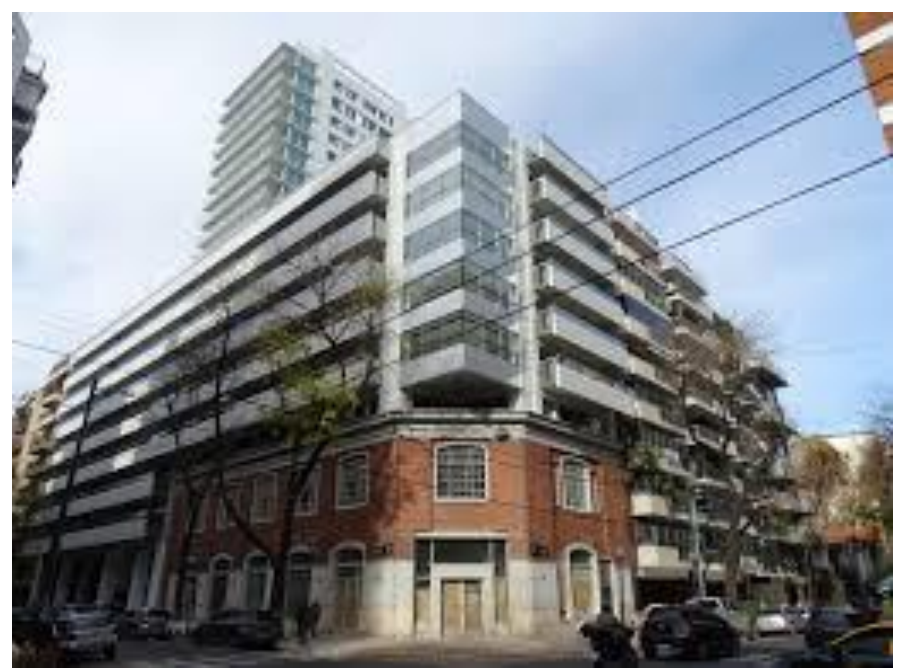

Figura 25. Vista del edificio terminado

Cuando esto no es posible, como en el caso presentado, es esencial compatibilizar el proyecto a construir con las estructuras de sostenimiento provisorias y una planificación muy cuidadosa de la secuencia de los trabajos.

En el trabajo presentado se recurre a una solución singular, esto es la utilización de la estructura de la edificación existente como estructura provisoria lo que simplifica enormemente las tareas, y las etapas de transferencias de cargas. disminuyendo las posibles afectaciones al muro.

Las demoliciones que se realizan, necesarias para desarrollar las nuevas obras, hacen perder rigidez a la estructura existente en su carácter de provisoria, por lo que se presentan soluciones adoptadas para mantener la estabilidad y la rigidez, Para poder realizar las excavaciones por debajo de la construcción original, se reemplazan las bases por pilotes excavados y vigas de transición para recibir las cargas de las columnas existentes, liberando así el área de trabajo.

El proceso constructivo adoptado plasmado en un plan de trabajos ordenado y minucioso en sus etapas y detalles, ejecutado con un control riguroso resultaron adecuados para se cumplieran los objetivos de la preservación del muro sin ningún tipo de afectación, integrándolo a la nueva construcción. 